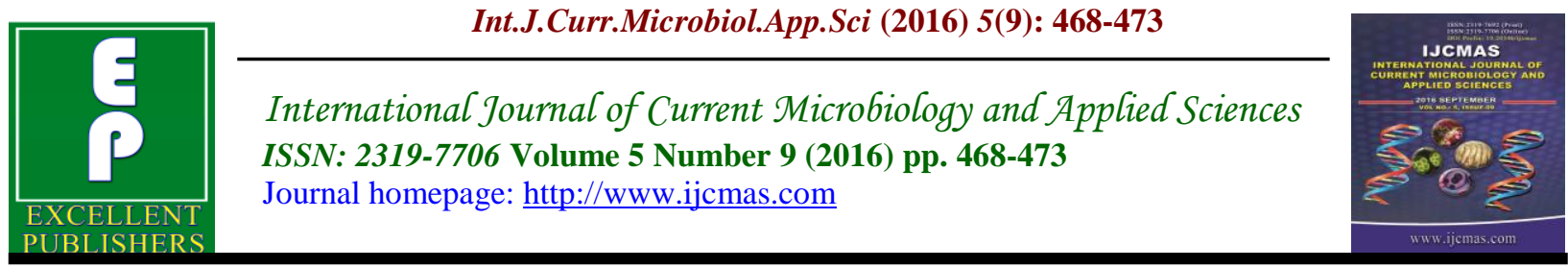

Original Research Article

http://dx.doi.org/10.20546/ijcmas.2016.509.051

\title{
Tinea cruris and Tinea genitalis due to Trichophyton interdigitale in and around Muzaffarnagar (Western UP), India: Possibly an Outbreak
}

\author{
Thakur Rameshwari ${ }^{1 *}$, Kushwaha Pragya ${ }^{2}$, Kumar Harish $^{3}$ and Paramjit Singh ${ }^{4}$ \\ Department of Microbiology, Muzaffarnagar Medical College, Opp: Begrajpur Industrial area, \\ Meerut Road, Muzaffarnagar (U.P.), India \\ *Corresponding author
}

Keywords

Trichophyton interdigitale,

Tinea cruris,

Tinea genitalis.

Dematophytes.

\section{Article Info}

Accepted:

18 August 2016

Available Online:

10 September 2016

\section{A B S T R A C T}

Identification of dematophyte causing Tinea cruris and Tinea genitalis by culture, biochemical and morphological characteristics in 130 cases during past six months, from February1 to July30, 2016. Patients suffering from tinea cruris or tinea genitalis were scraped from the affected area after cleaning the part with $70 \%$ alcohol. Potassium hydroxide mounts (KOH 20\%) were prepared for microscopy to see the fungal elements. Cultures were set up to study the morphological characteristics and for performing the biochemical tests and in vitro hair perforation test. All one hundred and thirty isolates were positive for microscopy and culture and were identified as Trichophyton interdigitale (former Trichophyton mentagrophyte) T. interdigitale was found to be the only species responsible for all the cases of tinea infection. More males were affected than females. Few single females were suffering from the tinea infection. Most of the married females, who had Tinea cruris or Tinea genitalis, gave the history of their spouses having acquired the infection first. Some single males who had Tinea genitalis, gave the history of having had sex with commercial sex worker. Two male patients gave the history of sharing the loincloth for wrestling. Few patients also had tinea corporis and tinea faciei. To avoid the spread of infection, it is important to identify the pathogen and start the treatment at the earliest.

\section{Introduction}

Tinea corporis and Tinea cruris are superficial dermatophyte infections and occur all over the world, but more common in tropical countries. Commonly, this is called ringworm. In Tinea cruris, there is dermatophyte infection of groin, but genitalia are usually spared even if the infection is extensive, whereas in Tinea genitalis, scrotum and penis are affected (Figure1) and in females, labia majora and mons pubis are also involved (Figure 2). Until now, Trichophyton rubrumhas been the most frequently isolated species (La Touche, 1967; Kumar et al., 1981; Pillai et al., 1975; Dekio et al., 1990) followed byTrichophyton mentagrophyte, Epidermophyton floccosum and Trichophyton verrucosum (La Touche, 1967). 
Spread of infection can be human to human, from animals and from soil.

Surprisingly, we started receiving many cases of Tinea cruris and Tinea genitalis all due to Trichophyton interdigitaleearly this year. Majority of the cases gave history of having had Tinea cruris/Tinea genitalis prior to tinea infection of other parts of the body. Non-had concomitant infection such as Tinea pedis or Tinea unguium. In this study, the patients gave varied history about how they got infected. Very few gave history of sexual contact in cases of Tinea cruris or Tinea genitalis. Two patients gave history of sharing langota (loin Cloth) used for wrestling in India.

\section{Patients and Methods}

During the past six months starting from Feb1, 2016to July 30, 2016, 130patients either with Tinea cruris or Tinea genitalis were enrolled for the study.

Only those patients with Tinea cruris or with Tinea genitalis were enrolled, who were not on antifungal drugs for past 4 weeks and lesions were of less than six months duration and in the age group 15-70 years.

Skin scrapings were taken from the affected areas in groin or from genital lesions if involved after thoroughly cleaning the lesions with $70 \%$ alcohol. Scrapings were also taken from any concomitant lesion elsewhere, e.g. toe nail, tenia pedis ortenia corporis if any and was processed and cultured separately in order to differentiate between autoinfection or mixed infection.

All the samples were examined for fungal elements in $\mathrm{KOH} 20 \%$ mount under high power of the microscope for fungal hyphae. Sabouraud's dextrose agar supplemented with cycloheximide and chloramphenicol was used and plates were incubated at $30^{\circ} \mathrm{C}$. Both positive and negative samples were inoculated on Sabouraud's Cycloheximide Chloramphenicol Agar (HI Media). Culture positive plates were seen for colony characteristics both on surface and reverse (Figure 3 and 5). Lacto phenol Cotton Blue (LCB) preparations by teased mount method and scotch tape method were prepared to study the microscopic structures in details (Figure4 and 6). Urease test and in vitro hair perforation tests were performed for the confirmation of Trichophyton interdigitale species. Also, other standard tests needed for the identification of dermatophytes were performed according to the description given in various textbooks, manuals, and journals (Nenoff et al., 2014; Rippon, 1988; Kwon-Chung et al., 1992; Larone, 2002; Vena et al., 2012).

Our study has limitation, because of the lack of molecular typing. It is impossible to differentiate between anthropophilic and zoophilic strains of $T$. interdigitale, because morphological differentiation by classical microscopic and biochemical methods is often problematic even in the hands of experienced mycologist.

\section{Clinical Results}

Most of the patients with Tinea cruris presented with extensive annular scaly lesions with central clearing. In male patients with Tinea genitalis along with the involvement of groin, there were scaly lesions on penis and scrotum. In female patients of Tinea genitalis, besides lesions in groin, there were erythematous, annular scaly lesions on mons pubis and labia majora.Non of our patients either with Tinea cruris or Tinea genitalis had pustular lesions or involvement of inguinal lymph nodes.

Out of a total 130 patients enrolled (84 males and 46 females), Tinea cruris was 
more common in the age group 26-35 year both in males $(\mathrm{n}=18[21.428 \%])$ and in females 14 (30.434\%) Tinea genitalis was also found to be more common in the same age group: males $(n=10$ [11.9047\%]) and in females $(n=4[8.695 \%]) \quad($ Table 1$)$. There were 7 couples in different age groups, who had Tinea cruris.

\section{Mycological Results}

Intrestingly, $T$. interdigitale was isolated from all the cases. Out of 100 cases with Tinea cruris, white cottony growth was seen in $(\mathrm{n}=75[75 \%])$, mixedi.e. partly granular and partly cottony in $(\mathrm{n}=22[22 \%])$ and granular in $(\mathrm{n}=3[3 \%])$.

Isolates from Tinea genitalis cases also had varied colony appearanceand out of 30 cases: White cottony was seen in $(n=10$ [33.33\%]), Mixed appearance $(n=14[46.66 \%])$ and granular $(n=6[20 \%])$.

\section{Discussion}

A recent report of tourists from Switzerland having visited South East Asia and having protected sex with commercial sex workers and later suffered from severe inflammatory Tinea genitalis due to $T$. interdigitale. Some of them even required hospitalization on their return (Luchsinger et al., 2015). Tinea genitalis has been reported from India from time to time in isolated reports (Pandey et al., 1981; Vora et al., 1994; Mukopadhyay, 2005). It is more common in hot and humid climate of tropics.

Table.1

\begin{tabular}{|c|c|c|c|c|}
\hline \multirow[t]{2}{*}{ Age in years } & \multicolumn{2}{|c|}{ Tinea cruris } & \multicolumn{2}{|c|}{$\begin{array}{l}\text { Tinea genitalis with } \\
\quad \text { Tinea cruris }\end{array}$} \\
\hline & Male & Female & Male & Female \\
\hline $15-25$ & $\begin{array}{c}32 \\
(38.096 \%)\end{array}$ & $\begin{array}{c}8 \\
(17.391 \%)\end{array}$ & $\begin{array}{c}7 \\
(8.333 \%)\end{array}$ & $\begin{array}{c}3 \\
(6.5217 \%)\end{array}$ \\
\hline $26-35$ & $\begin{array}{c}18 \\
(21.428 \%)\end{array}$ & $\begin{array}{c}14 \\
(30.434 \%)\end{array}$ & $\begin{array}{c}10 \\
(11.9047 \%)\end{array}$ & $\begin{array}{c}4 \\
(8.695 \%)\end{array}$ \\
\hline $36-45$ & $\begin{array}{c}7 \\
(8.333 \%)\end{array}$ & $\begin{array}{c}4 \\
(8.695 \%)\end{array}$ & $\begin{array}{c}3 \\
(3.571 \%)\end{array}$ & $\begin{array}{c}3 \\
(6.5217 \%)\end{array}$ \\
\hline $46-55$ & $\begin{array}{c}7 \\
(8.333 \%) \\
\end{array}$ & $\begin{array}{c}10 \\
(21.739 \%) \\
\end{array}$ & - & - \\
\hline Total & $\begin{array}{c}64 \\
(76.190 \%)\end{array}$ & $36(78.260 \%)$ & $\begin{array}{c}20 \\
(23.808 \%)\end{array}$ & $\begin{array}{c}10 \\
(21.739 \%)\end{array}$ \\
\hline
\end{tabular}

Fig.1 Tinea genitalis (Annular scaly lesions with central clearing and scaly lesions of penis and scrotum

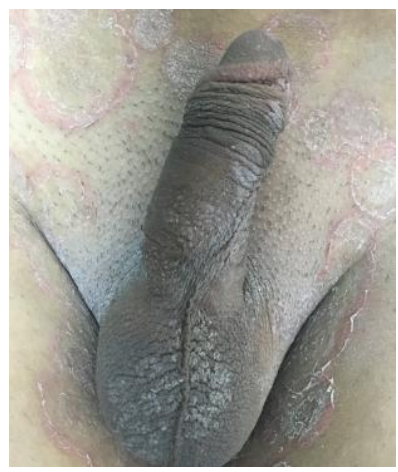


Fig.2 Tinea genitalis (Extensive scaly lesions of groin along with involvement of mons pubis and labia majora.

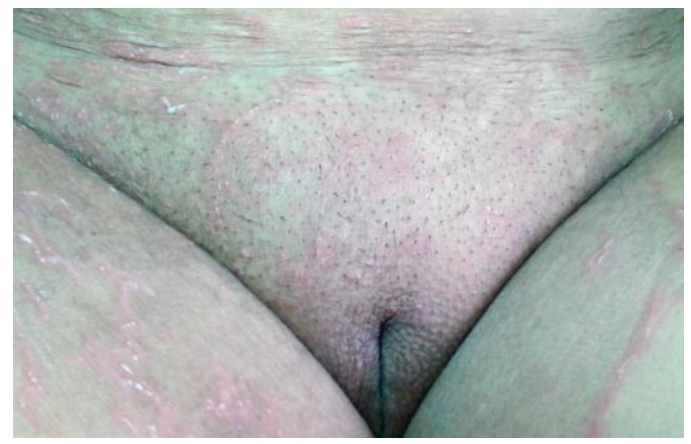

Fig.3 Cottony growth of T. interdigitale

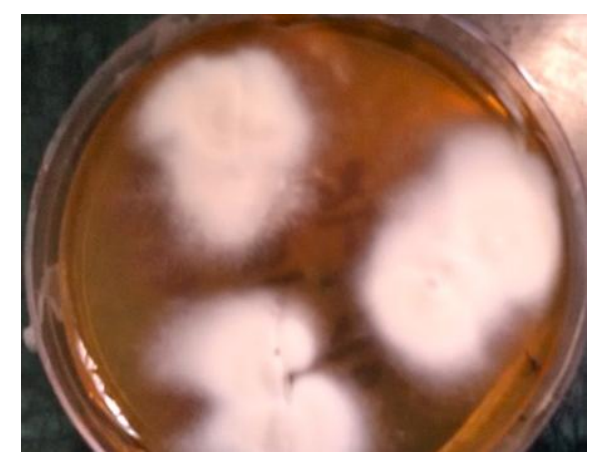

Fig.4 LCB mount

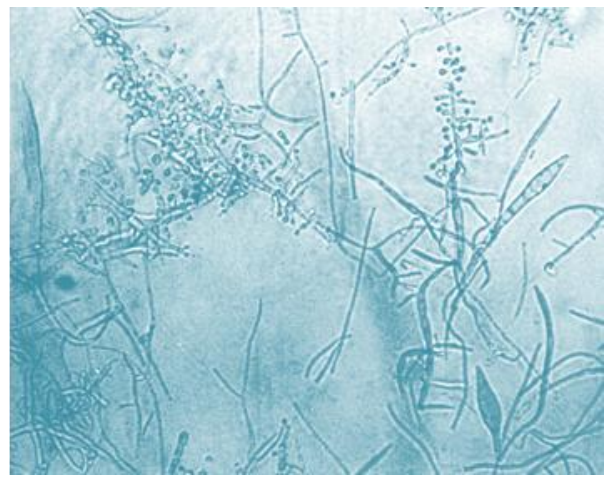

Fig.5 Powdery growth of T.interdigitale (Zoophilic strain)

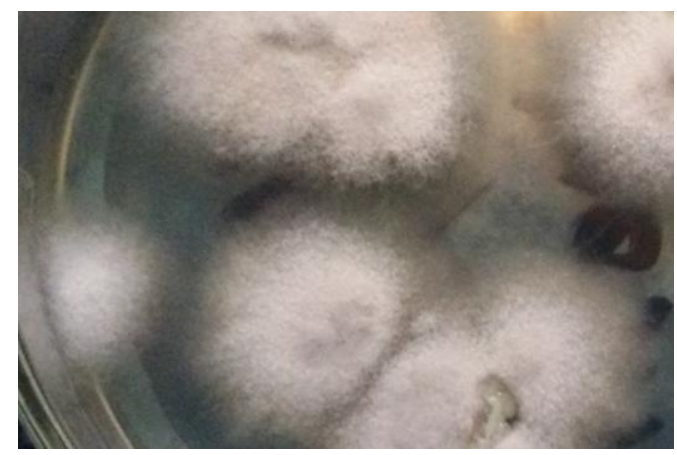


Fig.6 LCB mount (Abundant sporulation)

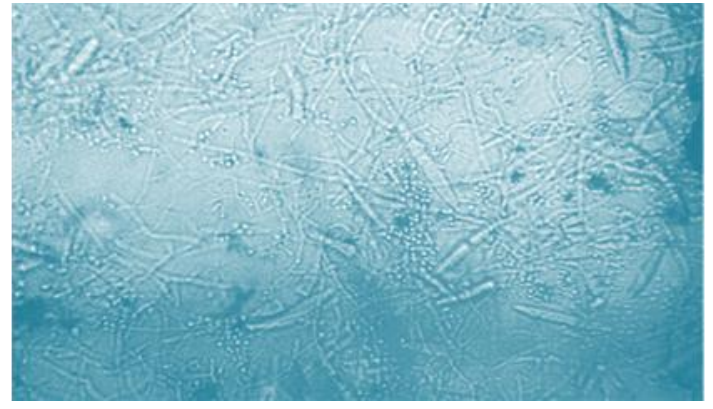

In a study by Kumar et al., (1981) they found $1 \%$ genital involvement in 2200 cases of dermatophyte infections. In another study by Pandey et al., 20\% patients with Tinea cruris also had genital involvement (Pandey et al., 1981).

Few male patients gave history of having had sex with commercial sex workers. One of the male patients gave history of being homosexual and had genital molluscum contagiosum. In case of female patients, especially married ones gave the history of their spouses suffering from Tinea cruris.

In one study of nine cases of Tinea genitalis by Romano et al from Italy (Romano et al., 2005), five cases of Tinea genitalis were due to $T$. rubrum, two due to E. flocossum and two due to $T$. interdigitale. There is no mention of sexual transmission, but concurrent infections like Tinea unguium of toenail, tinea pedis and tinea barbae is mentioned. Tinea genitalis and Tinea cruris can be transmitted sexually as well as by other means and it can be caused by both zoophilic and anthropophilic dermatophytes. None of our patients had highly inflammatory and pustular lesions, which goes in favour of infectionsbeing caused by anthropophilic strain of $T$. interdigitale. Also, lesions cleared after two weeks of treatment with Terbinafine $250 \mathrm{mg}$ once a day.
In conclusion, all cases of Tinea cruris should be examined carefully for tinea ganitalis. Though Tinea genitalis doesn't qualified to be called sexually transmitted infection, but Tinea cruris and Tinea genitalis can actually be transmitted sexually. Condoms have no role in the prevention of transmission of the infection. Lesions on the genital area should be looked for carefully, because it may point at the promiscuous sexual behaviour of the person and can help in diagnosing other sexually transmitted infections.

\section{References}

Dekio, S., Qin, L., Kawasaki, Y. et al. 1990. Tinea of scrotum: report of a case presenting as lichenified plaques. J. Dermatol., 17: 448-51.

Kumar, B., Talwar, P., Kaur, S. 1981. Penile tinea. Mycopathologia, 75: 169-72.

Kwon-Chung, K.J., Bennett, J.E. 1992. Dermatophytoses (Ringworm, Tinea, Dermatomycoses). In: Medical Mycology. 2nd ed. Philadelphia, PA: Lea and Febige, 105-170.

La Touche, C.J. 1967. Scrotal dermatophytosis. Br. J. Dermatol., 79: 339-44.

Larone, D.H. 2002. Dermatophytes. In: Medically Important Fungi: A Guide to Identification. 4th ed. Washington DC: American Society for Microbio- 
logy (ASM) Press, 229-253.

Luchsinger, I., Bosshard, P.P., Kasper, R.S., Reinhardt, D., Lautenschlager, S. 2015. Tinea genitalis: a new entity of sexuality transmitted infection? Case series and review of literature. Sex Transm. Infect., 0: 1-4.

Mukopadhyay, A.K. 2005. Trichophyton rubrum infection of prepuce. Indian $J$ Dermatol. Venerol. Leprol., 71: 130131.

Nenoff, P., Krüger, C., Schaller, J., et al. Mycology - an update part 2: dermatomycoses: clinical picture and diagnostics. J. Dtsch. Dermatol. Ges., 12: 749-77.

Pandey, S.S., Chandra, S., Guha, P.K. et al. 1981. Dermatophyte infection of penis; association with particular undergarments. Int. J. Dermatol., 20: 112-114.

Pandey, S.S., Chandra, S., Guha, P.K. et al. 1981. Dermatophyte infection of the penis; association with a particular undergarment. Int. J. Dermatol., 20: 112-114.
Pillai, K.G., Singh, G., Sharma, B.M. 1975. Trichophyton rubrum infection of the penis. Dermatologica, 150: 252-4.

Rippon, J.W. 1988. Dermatophytosis and Dermatomycosis. In: Medical Mycology: The Pathogenic Fungi and Pathogenic Actinomycetes. 3rd ed. Philadelphia, PA: Saunders, 169-275.

Romano, C., Ghilardi, A. and Papini, Manuela. 2005. Nine male cases of Tinea genitalis. Mycoses, 48: 202-204.

Vena, G.A., Chieco, P., Posa, F., Garofalo, A., Bosco, A., Cassano, N. 2012. Epidemiology of dermatophytoses: retrospective analysis from 2005 to 2010 and comparison with previous data from 1975. New Microbiol., 35(2): 207-213.

Vora, N.S., Mukopadhyay, A.K. 1994. Incidence of dermatophytosis of penis and scrotum. Indian J. Dermatol. Venerol. Lepro., 60: 89-91.

Weitzman, I., Summerbell, R.C. 1995. The Dermatophytes. Clin. Microbiol. Rev., 8(2): 240-259.

\section{How to cite this article:}

Thakur Rameshwari, Kushwaha Pragya, Kumar Harish and Paramjit Singh. 2016. Tinea cruris and Tinea genitalis due to Trichophyton interdigitale in and around Muzaffarnagar (Western UP), India: Possibly an Outbreak. Int.J.Curr.Microbiol.App.Sci. 5(9): 468-473. doi: http://dx.doi.org/10.20546/ijcmas.2016.509.051 